Article

\title{
Electrochemical Gating in Scanning Electrochemical Microscopy
}

Pivi Ahonen, Virginia Ruiz, Kysti Kontturi, Peter Liljeroth, and Bernadette M. Quinn

J. Phys. Chem. C, 2008, 112 (7), 2724-2728 • DOI: 10.1021/jp0776513

Downloaded from http://pubs.acs.org on January 14, 2009

\section{More About This Article}

Additional resources and features associated with this article are available within the HTML version:

- Supporting Information

- $\quad$ Links to the 1 articles that cite this article, as of the time of this article download

- $\quad$ Access to high resolution figures

- $\quad$ Links to articles and content related to this article

- Copyright permission to reproduce figures and/or text from this article

\section{View the Full Text HTML}




\title{
Electrochemical Gating in Scanning Electrochemical Microscopy
}

\author{
Päivi Ahonen, ${ }^{\dagger}$ Virginia Ruiz, ${ }^{\ddagger}$ Kyösti Kontturi, ${ }^{\dagger}$ Peter Liljeroth, $, \varangle, \S, \|$ and \\ Bernadette M. Quinn*, $\dagger$ \\ Laboratory of Physical Chemistry and Electrochemistry, Helsinki University of Technology, P.O. Box 6100, \\ FIN-02015 HUT, Finland, Department of Chemistry, University of Burgos, Pza. Misael Bañuelos, s/n, \\ Burgos 09001, Spain, and Condensed Matter and Interfaces, Debye Institute, University of Utrecht, \\ P.O. Box 80000, 3508 TA Utrecht, the Netherlands
}

Received: September 23, 2007; In Final Form: November 19, 2007

\begin{abstract}
We demonstrate that scanning electrochemical microscopy (SECM) can be used to determine the conductivity of nanoparticle assemblies as a function of assembly potential. In contrast to conventional electron transport measurements, this method is unique in that electrical connection to the film is not required. The electrochemical potential of the assembly is set through the Nernst equation by the redox mediator present in solution in analogy to the gate electrode in traditional three-terminal transport experiments. In this proof-of-concept report, we show that the conductance of an assembly of small gold nanocrystals (NCs), so-called monolayer-protected clusters (MPCs), is strongly dependent on the electrochemical potential. This is due to the sub-attofarad capacitance of the MPC cores that gives rise to single-electron charging effects at room temperature.
\end{abstract}

\section{Introduction}

Scanning electrochemical microscopy (SECM) has matured into a powerful tool to study diverse electrochemical systems. ${ }^{1}$ In particular, SECM has been used to probe lateral electron transport in thin nanocrystal (NC) or conductive polymer films. ${ }^{2}$ SECM provides a local, noninvasive probe for the sample conductivity with the added benefit of not having to contact the sample externally. ${ }^{2 \mathrm{~d}, 3}$ This avoids problems with the contact resistance between the film and the electrical contacts and the resulting ambiguity in the film potential. With SECM, the film potential is fixed by the equilibrium potential of the redox mediator used through the Nernst equation. ${ }^{2 \mathrm{~d}}$ In previous SECM studies, a single mediator concentration ratio was used, implying that sample conductivity was only probed at one particular value of substrate electrochemical potential. ${ }^{2}$ Here, we precisely vary the electrochemical potential of a thin film of hexanethiolate protected gold nanoparticles by controlling the ratio of the two forms of the solution redox mediator. This allows us to extract the conductivity of the sample as a function of the electrochemical potential. The method is conceptually similar to electrochemical gating of semiconducting nanoparticle films and carbon nanotubes where a reference electrode immersed in an electrolyte solution was used in place of a conventional back gate to control the electrochemical potential. ${ }^{4}$ In the SECM experiment (Figure 1), the heterogeneous electrochemical reaction between the solution redox mediator and the NC film acts as the source and the drain, while tuning the electrochemical potential through the choice and concentration ratio of the redox couple is analogous to gating in the usual three-terminal measurements.

Because nanocrystals have been proposed as potential building blocks for future nanoscale devices, there is considerable

* Corresponding authors. E-mail: P.Liljeroth@phys.uu.nl (P.L.) and bquinn@iki.fi (B.M.Q.).

Helsinki University of Technology.

$\doteqdot$ University of Burgos.

$\S$ University of Utrecht.

" Present Address: IBM Zurich Research Laboratory, 8803 Rüschlikon, Switzerland.
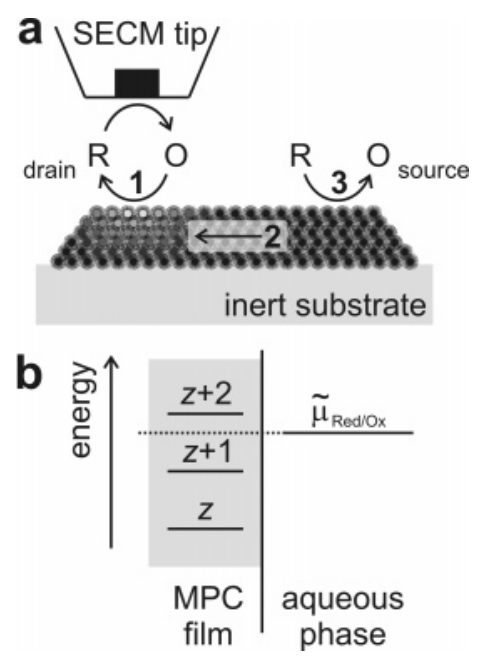

Figure 1. (a) Schematic of the experimental setup. The solution redox mediator is oxidized (reduced) at the SECM tip and reduced (oxidized) at the MPC film (process 1). This establishes an electrochemical potential gradient in the film, which is the driving force for lateral electron transport (process 2). The electrons are supplied by the reverse reaction between the MPCs and the solution redox couple outside the tip-substrate gap (process 3). In analogy with electrochemical gating, the electron transfer reaction between the solution redox mediator and the MPC film acts as the source (process 3 ) and the drain (process 1). (b) Energy level diagram of the experiment. The equilibrium electrochemical potential in the film is set by the nature (standard potential) of the redox couple and the concentration ratio of the oxidized to reduced species.

interest in their electronic properties. ${ }^{5}$ Particles of the type used in this study, metallic particles coated with an organic shell, so-called monolayer protected clusters (MPCs) are of particular interest due to their room-temperature quantized charging. $5 \mathrm{~b}, \mathrm{c}$ MPCs dispersed in a solution can be considered as diffusing nanoelectrodes ${ }^{6}$ and as conventional multivalent redox species in electrochemical experiments, ${ }^{5 c, 7}$ while films of MPCs have been proposed for sensing applications. ${ }^{2 \mathrm{c}, 8}$ The electron transport properties of MPC assemblies are determined by the core size 
and dispersity, the length and saturation of the protecting ligands and the interparticle separation. ${ }^{9}$ Murray and co-workers have demonstrated that for MPC films electron transport occurs by a bimolecular, electron self-exchange reaction, whose rate is controlled by the tunneling barrier due to the alkanethiolate ligands between the metal cores and the charge state, $z$, of the MPC cores. ${ }^{8 \mathrm{~m}}$ For mixtures of particles with $z=0$ and $z=1$, the conductivity was reported to be proportional to the product $\left[\mathrm{MPC}^{0}\right]\left[\mathrm{MPC}^{+}\right]$and goes through a maximum when the concentrations are equal. ${ }^{8 \mathrm{~m}}$ This is due to Coulomb blockade, which gives rise to periodic metal-insulator transitions at integer values of the average charge state of the particles. ${ }^{10}$ All reported data thus far has been obtained using techniques where an electrical connection to the film is a prerequisite to measuring the conductivity. ${ }^{8 \mathrm{a}-\mathrm{c}, \mathrm{e}-\mathrm{g}, \mathrm{k}, \mathrm{m}}$ This has been achieved by dropcasting the particles onto either a metal electrode for conventional electrochemical experiments such as voltammetry at the rotating disk electrode or an interdigitated array electrode for solid-state conductivity measurements. In the latter case, the $\left[\mathrm{MPC}^{0}\right]$ to $\left[\mathrm{MPC}^{+}\right]$ratio was controlled by ex situ chemical oxidation of the particles prior to drop-casting the film. ${ }^{8 m, 11}$ The effect of pinholes in the film, counterion permeation into thick films, and the contact resistance between the film and the electrode are all factors that influence the extracted film conductivity. In the present experiments, the film is drop-cast onto an insulating substrate and the measured SECM response is due solely to the film and not an underlying metal electrode. In this proof-of-principle report, we show that we can measure the conductance of the film as a function of the electrochemical potential without actually connecting it to an external power source.

\section{Experimental Section}

Chemicals. Ferrocene methanol (FcMeOH) (97\%, Aldrich), ferrocenylmethyl trimethylammonium iodide (FcTMAI) (Alfa Aesar), $\mathrm{LiCl}$ (p.a., Riedel-de Haën), and $\mathrm{Na}_{2} \mathrm{~S}_{2} \mathrm{O}_{8}$ (p.a., Fluka) were used as received. All solutions were prepared with MQ water. All other chemicals were of the highest available commercial purity and were used as received. Because iodide is corrosive to gold, it was replaced by $\mathrm{PF}_{6}^{-}$by metathesis of FcTMAI with $\mathrm{NH}_{4} \mathrm{PF}_{6}$ and all solutions were prepared with this salt. Hexanethiolate MPCs were synthesized according to a literature procedure known to yield particles of small core diameter. $^{7 \mathrm{c}, \mathrm{e}}$ The synthesis yields particles with a dominant population of monodisperse cores of average radius of $0.81 \mathrm{~nm}$, estimated from electrochemical measurements using the simple concentric sphere capacitor model to describe the MPC capacitance. The particles were assigned $\mathrm{Au}_{\sim 147}$ based on previous reports. ${ }^{5 \mathrm{c}, 7 \mathrm{~d}, \mathrm{e}}$

The redox couples, ferrocene methanol $\left(\mathrm{FcMeOH}^{0 /+}\right)$, and ferrocene trimethyammonium $\left(\mathrm{FcTMA}^{+/ 2+}\right)$ were used as the aqueous redox mediators in the SECM experiments. Because oxidized forms $(\mathrm{Ox})$ of the redox couples are not readily commercially available, they were prepared by chemical oxidation of freshly prepared solutions of the ferrocene derivatives (Red) with $\mathrm{Na}_{2} \mathrm{~S}_{2} \mathrm{O}_{8} .{ }^{12}$ The reaction is as follows:

$$
2 \mathrm{Fc}+\mathrm{S}_{2} \mathrm{O}_{8}^{2-} \rightarrow 2 \mathrm{Fc}^{+}+2 \mathrm{SO}_{4}^{2-}
$$

The protocol is described in the Supporting Information. Cyclic voltammetry (CV) at a Pt microelectrode was used to estimate the concentrations of the Ox and Red in solution. The resulting Ox solution was mixed with Red solutions in varying proportions to generate solutions with a controlled ratio of oxidized to reduced species for the SECM experiments.

SECM Experiments. The SECM substrate was prepared by pipetting a couple of drops of a concentrated chloroform solution of the MPCs on freshly cleaned microscope glass slides. The experiments were carried out with a commercial SECM $(\mathrm{CH}$ Instruments, US) using $10 \mu \mathrm{m}$ Pt microelectrodes (CH Instruments, US) with a known $R_{\mathrm{G}}$ (ratio of the radii of the insulating sheath and the electrode) as the SECM tip. An $\mathrm{Ag} / \mathrm{AgCl}$ wire served as both counter electrode and quasi-reference electrode (QRE). SECM approach curves at an approach rate of $1 \mu \mathrm{m}$ $\mathrm{s}^{-1}$ were obtained by biasing the tip at the diffusion-limited potential for the oxidation/reduction of the redox mediator $\left(\mathrm{FcMeOH}^{0 /+}\right.$ and $\left.\mathrm{FcTMA}^{+/ 2+}\right)$ and recording the tip current as a function of distance $l$ to the substrate. $\mathrm{LiCl}(0.1 \mathrm{M})$ was used throughout as the supporting electrolyte. A series of approach curves was measured by changing the total mediator concentration, $c^{\text {b}}$, by dilution with $0.1 \mathrm{M} \mathrm{LiCl}$ thus keeping the dimensionless concentration ratio of the reduced form of the solution redox couple $C^{\mathrm{b}}\left(=c_{\mathrm{Red}}^{\mathrm{b}} /\left(c_{\mathrm{Red}}^{\mathrm{b}}+c_{\mathrm{Ox}}^{\mathrm{b}}\right)=c_{\mathrm{Red}}^{\mathrm{b}} / c^{\mathrm{b}}\right)$ constant where $c_{\mathrm{Red}}^{\mathrm{b}}$ and $c_{\mathrm{Ox}}^{\mathrm{b}}$ are the bulk concentrations of the reduced and oxidized species in solution and $c^{\mathrm{b}}$ is the total bulk concentration. The experimental protocol has been described in detail elsewhere. ${ }^{2 f}$

Before every approach curve, a $\mathrm{CV}$ of the redox mediator was recorded at the SECM tip. The value of $C^{\text {b }}$ was determined from the ratio of the limiting currents for mediator oxidation and reduction at the tip. The substrate potential was taken as the equilibrium potential of the mediator in solution and is determined by $c_{\mathrm{Red}}^{\mathrm{b}} / c_{\mathrm{Ox}}^{\mathrm{b}}$ through the Nernst equation. Because a QRE was used, the measured potential was corrected to the Ag/ $\mathrm{AgCl}$ using the measured half-wave potential, $E_{1 / 2}$, for the mediator as an internal reference assuming $E_{1 / 2}=E^{0^{\prime}}$ where $E^{0^{\prime}}$ $=0.21 \mathrm{~V}(\mathrm{FcMeOH})$ and $0.42 \mathrm{~V}\left(\mathrm{FcTMA}^{+}\right)$versus $\mathrm{Ag} / \mathrm{AgCl}$. The substrate potential could be varied between $137 \mathrm{mV}$ and $460 \mathrm{mV}$ versus $\mathrm{Ag} / \mathrm{AgCl}$ with the chosen mediators. The corresponding corrections are given in the Supporting Information.

\section{Results and Discussion}

As illustrated in Figure 1a, the solution redox mediator is oxidized (reduced) at the SECM tip and re-reduced (reoxidized) at the MPC film. These local changes set up an electrochemical potential gradient in the MPC film, which is the driving force for lateral electron transport. The electrons are injected into the film by the same redox couple outside the tip-substrate gap. Using a model that has been described previously, ${ }^{2 \mathrm{~d}}$ the experimental approach curves (tip current vs tip-substrate separation) can be fitted to yield a quantitative estimate on the electrical conductance of the MPC film.

With this model, electron transport in the film is described by eq 1 , in dimensionless radial coordinates, $R=r / r_{e}$, assuming Ohmic conduction and Gerischer model for electron transfer kinetics $^{2 \mathrm{~d}}$

$$
\frac{\partial^{2} \tilde{\mu}}{\partial R^{2}}+\frac{1}{R} \frac{\partial \tilde{\mu}}{\partial R}-\frac{K^{0}}{\Sigma}\left((1-C) e^{\tilde{\mu} / 2}-C e^{-\tilde{\mu} / 2}\right)=0
$$

where $\tilde{\mu}=\left(\mu-\mu^{0}\right) / k_{\mathrm{B}} T$ is the dimensionless electrochemical potential of the electrons in the film, $K^{0}=k^{0} r_{\mathrm{e}} / D$ is the dimensionless standard rate constant of the electron transfer reaction between the solution redox couple and the nanoparticle film $\left(r_{\mathrm{e}}\right.$ is the electrode radius and $D$ is the redox mediator diffusion coefficient), $C=c_{\text {Red }} /\left(c_{\text {Red }}^{\mathrm{b}}+c_{\mathrm{Ox}}^{\mathrm{b}}\right)=c_{\mathrm{Red}} / c^{\mathrm{b}}$ is the 

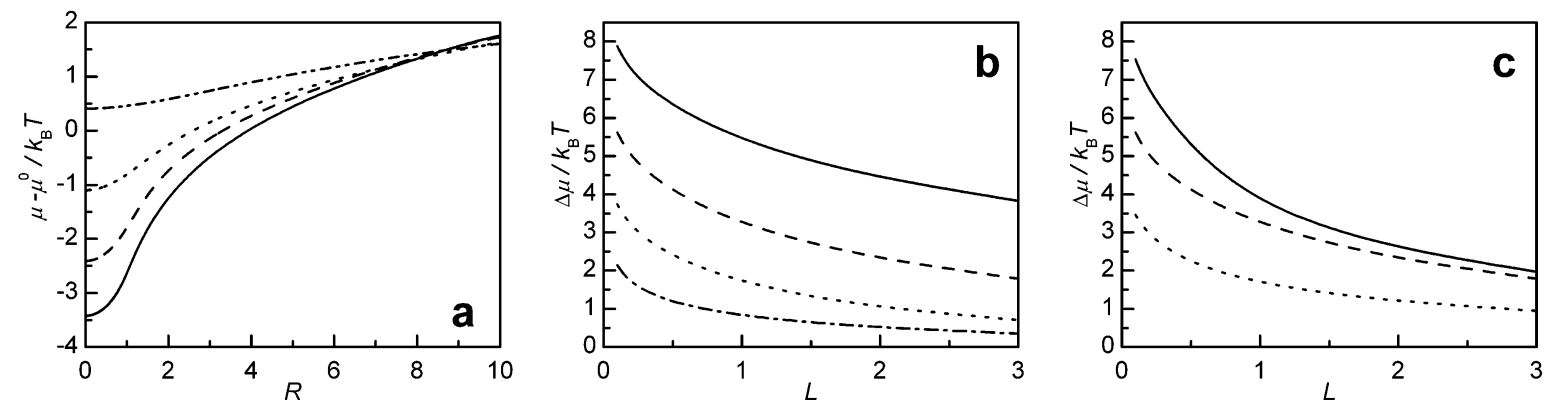

Figure 2. (a) Simulated electrochemical potential profiles in the units of $k_{\mathrm{B}} T$ in the MPC film at different SECM tip-substrate separations, $L$, from bottom to top 0.1 (solid line), 0.3 (dashed), 1.0 (dotted), and 3.0 (dash-dotted). Dimensionless conductivity $\Sigma=0.1$. (b) Simulated values for the electrochemical potential difference (in the units of $k_{\mathrm{B}} T$ ) directly under the SECM tip compared to the equilibrium value far from the tip-substrate gap (analogous to source-drain voltage difference) for different concentration ratios of the solution redox mediator: from top to bottom $C^{\mathrm{b}}=0.99$ (solid line), 0.9 (dashed), 0.5 (dotted), and 0.1 (dash-dotted). Dimensionless conductivity $\Sigma=0.1$. (c) Simulated values for the electrochemical potential difference (in the units of $k_{\mathrm{B}} T$ ) directly under the SECM tip compared to the equilibrium value far from the tip-substrate gap for different dimensionless conductivities of the film, from top to bottom $\Sigma=0.01$ (solid line), 0.1 (dashed), and 1.0 (dotted). $C^{\text {b }}=0.9$. Other parameters: $R_{\mathrm{G}}$ $=10, K^{0}=10$.

dimensionless concentration of the reduced form of the solution redox couple, and $\Sigma$ is the dimensionless conductivity in the film given by $\Sigma=\sigma k_{\mathrm{B}} T \Delta z /\left(e^{2} r_{\mathrm{e}} D c^{\mathrm{b}} N_{\mathrm{A}}\right)$, where $\sigma$ is conductivity, $\Delta z$ the thickness of the film, and other symbols have their usual meaning. Outside the tip-substrate gap, the electrochemical potential of the electrons in the $\mathrm{NC}$ film reaches an equilibrium value, $\tilde{\mu}_{\text {eq }}$, which is determined by the concentration ratio of the oxidized and reduced forms of the redox couple.

Using this model, families of simulated approach curves (SECM tip current as a function of the normalized tip-substrate distance $L=l / r_{\mathrm{e}}$ ) were generated using a commercially available finite element simulation package (Comsol Multiphysics, Comsol $\mathrm{Ab}, \mathrm{Sweden})$ for each $\tilde{\mu}_{\mathrm{eq}}$ over a wide range of $\Sigma$ values. As described in the previous publications, ${ }^{2 b, d}$ the so-called back diffusion (diffusion of the solution redox couple behind the plane of the electrode) was included in the model. Experimental curves were then compared with simulated curves, and the corresponding best fit was used to extract $\Sigma$. The film conductance can be obtained from the slope of the plot of $\Sigma$ versus the reciprocal total mediator concentration. We do not observe kinetic limitations with the redox mediators used in this study, and consequently all of the simulations were carried out with a high value of $K^{0}(=10)$ to give numerically undistinguishable results from $K^{0}=\infty$.

The equilibrium potential of the film is given simply by the Nernst equation

$$
E_{\mathrm{eq}}=E^{0}+R T / F \ln \left(c_{\mathrm{Ox}}^{\mathrm{b}} / c_{\mathrm{Red}}^{\mathrm{b}}\right)
$$

and can be tuned by varying either the redox couple (and thus the standard redox potential $E^{0}$ ) or the concentration ratio. However, when the SECM tip is brought to close proximity of the substrate, the local perturbation due to the redox reaction at the tip will result in an electrochemical potential gradient in the film. This gradient causes electron transport in the film in the radial direction and is analogous to the bias difference applied between the source and the drain electrodes in a traditional transport experiment. Electrochemical potential profiles (in the units of $k_{\mathrm{B}} T$ ) for different tip-substrate separations, $L$, are plotted in Figure 2a for a typical value of the dimensionless conductivity $\Sigma=0.1$. When the SECM tip is far from the film, the electrochemical potential in the film only changes slightly: the potential difference directly under the SECM tip compared to the equilibrium potential is $<2 k_{\mathrm{B}} T$ (corresponding to $<50 \mathrm{mV}$ at room temperature). The magnitude of the perturbation is increased the closer the tip is to the substrate, reaching values close to $6 k_{\mathrm{B}} T(\sim 150 \mathrm{mV})$ with these values of $C^{\text {b }}$ and $\Sigma$ (vide infra) at a tip-substrate distance of $L=0.1$, which is a typical closest distance that can be reached in experiments. The effect of varying $C^{\mathrm{b}}$ (for oxidation at the tip) on the electrochemical potential difference in the film is investigated in Figure 2b. It is clear that the concentration ratio has an effect on the magnitude of the perturbation with the values ranging up to $8 k_{\mathrm{B}} T$ for small $L$ and $C^{\mathrm{b}}$ close to 1 . Finally, Figure $2 \mathrm{c}$ shows the effect of the film conductivity ranging from poorly conducting (SECM response close to pure negative feedback, solid line) to conducting films (SECM response close to pure positive feedback, dotted line). These changes can be readily rationalized: in metals (infinite conductivity), an electrochemical potential gradient cannot exist and the less conductive the substrate, the larger the perturbation imposed by the local electrochemical reaction at the SECM tip.

The electrochemical potential profile generated by the SECM tip has important implications for the experiments. By assuming that the film behaves as an Ohmic conductor, we essentially average the film conductance over the difference of electrochemical potential directly under the SECM tip compared to the equilibrium value far from the tip-substrate gap. On the basis of the simulations shown above, the magnitude of this effect is typically of the order of $4 k_{\mathrm{B}} T$, corresponding to 100 $\mathrm{mV}$ at room temperature.

In order to validate the proposed method to control the applied potential, we recorded approach curves over a range of total concentration of the redox mediator $c^{\mathrm{b}}=c_{\mathrm{Ox}}^{\mathrm{b}}+c_{\text {Red }}^{\mathrm{b}}$ with $C^{\mathrm{b}}=$ $c_{\text {Red }}^{\mathrm{b}} / c^{\mathrm{b}}$ fixed to value 0.45 while either reducing (Figure $3 \mathrm{a}$ ) or oxidizing (Figure $3 b$ ) the solution redox mediator at the SECM tip. The observed feedback response in Figure 3 is a combination of the diffusion flux in the solution and the flux due to electron transport in the film. Because the diffusion flux is directly proportional to the total concentration of the redox mediator in solution, to observe electron transport the concentration has to be reduced sufficiently. At high redox couple concentrations, negative feedback due to hindered diffusion to the SECM tip is observed. As the concentration is reduced, the relative contribution of mediator regeneration due to electron transport in the film increases and deviation from purely negative feedback will be observed. This effect is captured by the dimensionless conductivity, $\Sigma$. The experimental approach curves shown in Figure 3 were fitted to the theory with only $\Sigma$ as a free parameter. The results are shown in the insets of Figure 3 , where the extracted $\Sigma$ values are plotted as a function of the inverse of the total concentration $c^{\text {b }}$ of the solution redox 

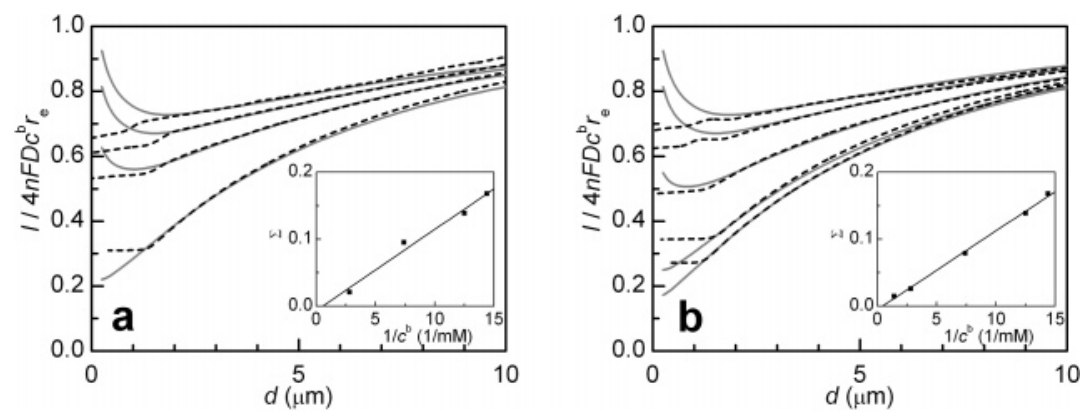

Figure 3. Experimental SECM approach curves (dotted lines) along with the fits to simulations (solid gray lines) for the same ratio of the reduced to oxidized forms of the solution redox mediator $\left(c_{\mathrm{Red}}^{\mathrm{b}} / c^{\mathrm{b}}=0.45\right)$ at different total concentrations corresponding to either reduction $(\mathrm{a})$ or oxidation (b) at the SECM tip. The parameters used in the measurements: (a) Tip potential $0.1 \mathrm{~V}$ corresponding to reduction of $\mathrm{FcMeOH}^{+}$at total concentrations of $69,80,136$, and $357 \mu \mathrm{M}$ (top to bottom). (b) Tip potential $0.25 \mathrm{~V}$ corresponding to oxidation of FcMeOH using concentrations $69,80,136,357$, and $701 \mu \mathrm{M}$ (top to bottom). Insets: Linear regression of the dimensionless conductivity $\Sigma$ as function of $1 / c^{\mathrm{b}}$. The resulting conductance $\sigma \Delta z$ is $1.58 \times 10^{-10} 1 / \Omega$ for the reduction reaction and $1.55 \times 10^{-10} 1 / \Omega$ for the oxidation of the mediator.

mediator. As expected, these plots are linear. Moreover, because the slope is proportional to the conductance of the film, we measure the same value irrespective whether oxidation or reduction is taking place at the tip. This also gives an experimental indication of the magnitude of the averaging process due to the electrochemical potential gradient in the film. We bias the film in a different direction from the equilibrium potential depending on whether oxidation or reduction reaction is occurring at the SECM tip and yet, we measure the same value for film conductance.

The equilibrium electrochemical potential of the film is set by the nature and the concentration ratio of the reduced and oxidized forms of the solution redox mediator, which is analogous to the gate electrode in traditional transport experiments. Thus, we next varied these parameters to probe how the film conductance depends on the electrochemical potential. The MPCs used in this study exhibit single-electron charging at room-temperature due to their small size and the associated subattofarad capacitance of the protecting thiolate monolayer. ${ }^{5 \mathrm{c}}$ This means that additional energy (charging energy) has to be overcome to change the charge state of the nanoparticles by one electron. This is illustrated schematically in Figure 1b, which shows how by tuning the electrochemical potential of the solution redox couple we can continuously tune the average charge state of the MPCs in the film, while the SECM measurements give an experimental handle on the corresponding film conductance. $\mathrm{FcMeOH}^{0 /+}$ and $\mathrm{FcTMA}^{+/ 2+}$ were chosen as redox mediators because both undergo reversible electron transfer with the MPCs. ${ }^{3,5}$ This avoids having to include the kinetic parameter $K^{0}$ in the fitting, and thus there is only a single free parameter in the model, the dimensionless conductivity $\Sigma$.

Consequently, by varying the concentrations of the oxidized and reduced forms of the solution redox couple and by using two different redox couples having differing standard potentials, we can access the conductance of the MPC film over a wide range of electrochemical potentials. The experimental protocol was repeated for a range of concentration ratios of both redox couples, and the dimensionless conductivity was extracted from the slope of the corresponding plots of $\Sigma$ versus the reciprocal mediator concentration.

The conductance values obtained are plotted versus film potential in Figure 4. It has two maxima with an overall variation of over a factor of 2 within the studied range of electrochemical potentials. This can be attributed to the room-temperature singleelectron charging of the MPCs: The conductance of the film is proportional to $n_{z}\left(1-n_{z}\right)$, where $n_{z}$ is the occupancy of a given charge state $z$. The maximum conductance is obtained at halfshell filling $n_{z}=1 / 2$, while the conductance assumes low values

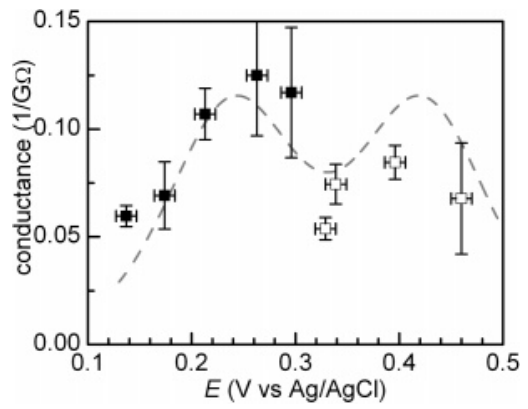

Figure 4. Conductance $\sigma \Delta z$ extracted from the fits to the SECM approach curves as a function of the equilibrium electrochemical potential of the MPC film. The redox couple was $\mathrm{FcMeOH}$ (filled squares) or FcTMA (open squares). The error bars correspond to the error in the slope of $\Sigma$ vs $1 / c^{\mathrm{b}}$ as estimated by linear regression analysis. Dashed gray line is a fit to a simple model where conductance is proportional to the product of $n(1-n)$, yielding $E_{\mathrm{c}}=185 \mathrm{meV}$.

at $n_{z}=0$ or 1 . To construct a simple model of this, we assume that the occupancy of a charge state $n_{z}$ is given by the Fermi distribution $n_{z}=1 /\left(1+\mathrm{e}^{\left(E_{\mathrm{eq}}-\mu_{z}\right) / w}\right)$ with the electrochemical potential of that state $\mu_{z}$. The width of the distribution $w$ is determined mainly by the temperature and inhomogeneous broadening due to the size-dispersion of the MPCs and the distribution in the charging energies due to differences in the local environment. ${ }^{10 a}$ The conductance of the assembly is then the sum over the different charge states $\sum_{z} n_{z}\left(1-n_{z}\right)$, where the electrochemical potentials of the different charge states are separated by the charging energy $E_{\mathrm{c}}$. The fit to this model for a sum of two charge states is shown by the dashed gray line in Figure 4. The data given in Figure 4 is representative of a number of measurements, and we qualitatively observe the same behavior in all cases with variations in the film conductance values due to the differing film thicknesses and small shifts in the positions of the electrochemical potential where the peaks occur.

The observation of maxima is in qualitative agreement with the reported dependence of film conductance on the degree of mixed valency for nominally identical MPCs. ${ }^{8 m, 11}$ The separation of the maxima in the conductance gives the charging energy of the MPCs in the film $(185 \mathrm{meV})$, which is lower than the value measured for MPC dispersed in an organic solvent $(260 \mathrm{meV}) .^{7 \mathrm{c}}$ The difference can be attributed to the different dielectric environment of the particles in the film or solution. ${ }^{13}$

This report contrasts with our recent determination of the conductivity of an MPC monolayer with a larger core radius (6.6 vs $1.7 \mathrm{~nm})$ where both redox mediators (FeMeOH and $\left.\mathrm{Fe}(\mathrm{CN}) 6^{3-/ 4-}\right)$ yielded comparable values of the conductivity 
despite the different standard potentials. ${ }^{2 \mathrm{~b}}$ This can be rationalized by the significantly decreased charging energy for electron injection into cores of larger radius. ${ }^{9 b}$ Variations in the conductivity due to the particle charging energy cannot be resolved at room temperature because the charging energy is comparable to $k_{\mathrm{B}} T$. $^{9 \mathrm{~b}}$

\section{Conclusions}

SECM enables a quantitative measurement of the potential dependence of electron transport in nanoparticle assemblies without requiring an external electrical connection to the assembly. This type of electrochemical gating avoids issues with the resistance between the film and the electrical contacts on conventional electron transport measurements and the resulting ambiguity in the film potential. We demonstrate that the conductance of MPC films is strongly dependent on the film electrochemical potential due to room-temperature singleelectron charging of the individual MPCs.

Acknowledgment. We acknowledge financial support from the Academy of Finland (Academy Research Fellow, B.M.Q.), Nederlandse Organisatie voor Wetenschappelijk Onderzoek (NWO/Chemical Sciences, Vidi-grant 700.56.423, P.L.), the Ministerio de Educación y Ciencia (Juan de la Cierva contract, V.R.) and European Commission under the PF6 programme (Design and functionality of non-linear electrochemical nanoscale devices, DYNAMO).

Supporting Information Available: Additional experimental details and results. This material is available free of charge via the Internet at http://pubs.acs.org.

\section{References and Notes}

(1) Bard, A. J.; Faulkner, L. R. Electrochemical Methods, Fundamentals and Applications, 2nd ed.; John Wiley \& Sons Inc.: New York, 2001.

(2) (a) Nicholson, P. G.; Ruiz, V.; Macpherson, J. V.; Unwin, P. R. Phys. Chem. Chem. Phys. 2006, 8, 5096. (b) Liljeroth, P.; Quinn, B. M. J. Am. Chem. Soc. 2006, 128, 4922. (c) Ruiz, V.; Nicholson, P. G.; Jollands, S.; Thomas, P. A.; Macpherson, J. V.; Unwin, P. R. J. Phys. Chem. B 2005, 109, 19335. (d) Liljeroth, P.; Vanmaekelbergh, D.; Ruiz, V.; Kontturi, K.; Jiang, H.; Kauppinen, E.; Quinn, B. M. J. Am. Chem. Soc. 2004, 126, 7126. (e) Zhang, J.; Barker, A. L.; Mandler, D.; Unwin, P. R. J. Am. Chem. Soc. 2003, 125, 9312. (f) Ruiz, V.; Liljeroth, P.; Quinn, B. M.; Kontturi, K. Nano Lett. 2003, 3, 1459. (g) Mandler, D.; Unwin, P. R. J. Phys. Chem. B 2003, 107, 407. (h) Liljeroth, P.; Quinn, B. M.; Ruiz, V.; Kontturi, K. Chem.
Commun. 2003, 1570. (i) Quinn, B. M.; Prieto, I.; Haram, S. K.; Bard, A. J. J. Phys. Chem. B 2001, 105, 7474.

(3) Whitworth, A. L.; Mandler, D.; Unwin, P. R. Phys. Chem. Chem. Phys. 2005, 7, 356.

(4) (a) Cronin, S. B.; Barnett, R.; Tinkham, M.; Chou, S. G.; Rabin, O.; Dresselhaus, M. S.; Swan, A. K.; Unlu, M. S.; Goldberg, B. B. Appl. Phys. Lett. 2004, 84, 2052. (b) Yu, D.; Wang, C. J.; Guyot-Sionnest, P. Science 2003, 300, 1277. (c) Roest, A. L.; Kelly, J. J.; Vanmaekelbergh, D.; Meulenkamp, E. A. Phys. Rev. Lett. 2002, 89, 036801.

(5) (a) Love, J. C.; Estroff, L. A.; Kriebel, J. K.; Nuzzo, R. G.; Whitesides, G. M. Chem. Rev. 2005, 105, 1103. (b) Daniel, M.-C.; Astruc, D. Chem. Rev. 2004, 104, 293. (c) Templeton, A. C.; Wuelfing, W. P.; Murray, R. W. Acc. Chem. Res. 2000, 33, 27.

(6) Pietron, J. J.; Hicks, J. F.; Murray, R. W. J. Am. Chem. Soc. 1999, $121,5565$.

(7) (a) Peterson, R. R.; Cliffel, D. E. Langmuir 2006, 22, 10307. (b) Georganopoulou, D. G.; Mirkin, M. V.; Murray, R. W. Nano Lett. 2004, 4 1763. (c) Quinn, B. M.; Liljeroth, P.; Ruiz, V.; Laaksonen, T.; Kontturi, K. J. Am. Chem. Soc. 2003, 125, 6644. (d) Miles, D. T.; Murray, R. W. Anal. Chem. 2003, 75, 1251. (e) Hicks, J. F.; Miles, D. T.; Murray, R. W. J. Am. Chem. Soc. 2002, 124, 13322. (f) Chen, S. W.; Murray, R. W.; Feldberg, S. W. J. Phys. Chem. B 1998, 102, 9898. (g) Chen, S. W.; Ingram, R. S.; Hostetler, M. J.; Pietron, J. J.; Murray, R. W.; Schaaff, T. G.; Khoury, J. T.; Alvarez, M. M.; Whetten, R. L. Science 1998, 280, 2098.

(8) (a) Wang, L. Y.; Shi, X. J.; Kariuki, N. N.; Schadt, M.; Wang, G. R.; Rendeng, Q.; Choi, J.; Luo, J.; Lu, S.; Zhong, C. J. J. Am. Chem. Soc. 2007, 129, 2161. (b) Rowe, M. P.; Steinecker, W. H.; Zellers, E. T. Anal. Chem. 2007, 79, 1164. (c) Krasteva, N.; Fogel, Y.; Bauer, R. E.; Mullen, K.; Matsuzawa, N.; Yasuda, A.; Vossmeyer, T. Adv. Funct. Mater. 2007, 17, 881. (d) Ibanez, F. J.; Gowrishetty, U.; Crain, M. M.; Walsh, K. M.; Zamborini, F. P. Anal. Chem. 2006, 78, 753. (e) Leopold, M. C.; Donkers, R. L.; Georganopoulou, D.; Fisher, M.; Zamborini, F. P.; Murray, R. W. Faraday Discuss. 2004, 125, 63. (f) Brennan, J. L.; Branham, M. R.; Hicks, J. F.; Osisek, A. J.; Donkers, R. L.; Georganopoulou, D. G.; Murray, R. W. Anal. Chem. 2004, 76, 5611. (g) Zamborini, F. P.; Smart, L. E.; Leopold, M. C.; Murray, R. W. Anal. Chim. Acta 2003, 496, 3. (h) Zamborini, F. P.; Leopold, M. C.; Hicks, J. F.; Kulesza, P. J.; Malik, M. A.; Murray, R. W. J. Am. Chem. Soc. 2002, 124, 8958. (i) Wuelfing, W. P.; Murray, R. W. J. Phys. Chem. B 2002, 106, 3139. (j) Hicks, J. F.; Young, S.-S.; Murray, R. W. Langmuir 2002, 18, 2288. (k) Hicks, J. F.; Zamborini, F. P.; Osisek, A.; Murray, R. W. J. Am. Chem. Soc. 2001, 123, 7048. (1) Zamborini, F. P.; Hicks, J. F.; Murray, R. W. J. Am. Chem. Soc. 2000, 122, 4514. (m) Wuelfing, W. P.; Green, S. J.; Pietron, J. J.; Cliffel, D. E.; Murray, R. W. J. Am. Chem. Soc. 2000, 122, 11465. (n) Templeton, A. C.; Zamborini, F. P.; Wuelfing, W. P.; Murray, R. W. Langmuir 2000, 16, 6682.

(9) (a) Markovich, G.; Collier, C. P.; Henrichs, S. E.; Remacle, F.; Levine, R. D.; Heath, J. R. Acc. Chem. Res. 1999, 32, 415. (b) Collier, C. P.; Vossmeyer, T.; Heath, J. R. Annu. Rev. Phys. Chem. 1998, 49, 371.

(10) (a) Chandler, R. E.; Houtepen, A. J.; Nelson, J.; Vanmaekelbergh, D. Phys. Rev. B 2007, 75, 085325. (b) Vanmaekelbergh, D.; Liljeroth, P. Chem. Soc. Rev. 2005, 34, 299.

(11) Choi, J. P.; Murray, R. W. J. Am. Chem. Soc. 2006, 128, 10496.

(12) Fan, F. R. F.; Bard, A. J. Science 1995, 267, 871.

(13) Garcia-Morales, V.; Mafe, S. J. Phys. Chem. C 2007, 111, 7242. 